\title{
Mutation of the Arg191 in FtsZ impairs cytokinetic abscission of Bacillus
}

\section{subtilis cells}

Funding source - The work is supported by a grant from Department of Science and Technology (DST, India) to DP.

Hemendra Pal Singh Dhaked ${ }^{1,+}$, Anusri Bhattacharya ${ }^{1,+}$, Saroj Yadav², Sarath Chandra Dantu $^{1}$, Ashutosh Kumar ${ }^{1}$, \& Dulal Panda ${ }^{1, *}$

${ }^{1}$ Department of Biosciences and Bioengineering, Indian Institute of Technology Bombay, Mumbai, 400076, India

${ }^{2}$ IITB-Monash Research Academy, Indian Institute of Technology Bombay, Mumbai, 400076, India

${ }^{+}$These authors contributed equally to this work

*Corresponding Author - Department of Biosciences and Bioengineering, Indian Institute of Technology Bombay, Powai, Mumbai 400076, India. Telephone: 91-22-2576-7838. Fax: 91-22-2572-3480. Email: panda@iitb.ac.in. 


\section{Supporting Figures}
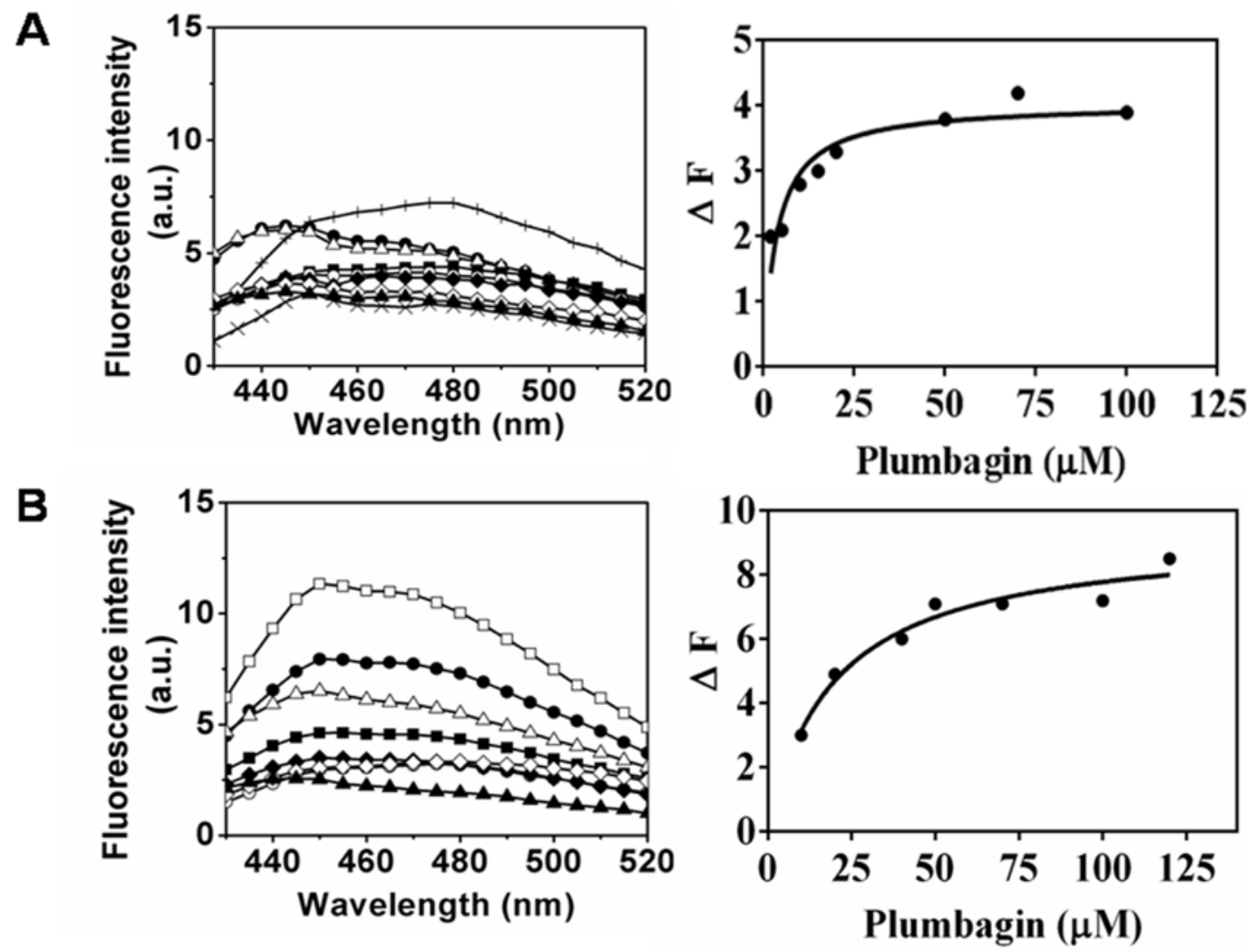

Fig. S1. Effects of plumbagin on the fluorescence intensity of ANS in the presence of WTFtsZ or with R191A-FtsZ. (A) WT-FtsZ $(2 \mu \mathrm{M})(+)$ was incubated with $2(\bullet), 5(\Delta), 10(\mathbf{})$, $15(\circ), 20(\diamond), 50(\diamond), 70(\Delta)$, and $100 \mu \mathrm{M}(\times)$ plumbagin at $25^{\circ} \mathrm{C}$ for $10 \mathrm{~min}$. (B) R191AFtsZ $(2 \mu \mathrm{M})(\square)$ was incubated with $10(\bullet), 20(\Delta), 40(\bullet), 50(\circ), 70(\diamond), 100(\diamond)$ and120 $\mu \mathrm{M}(\boldsymbol{\Delta})$ plumbagin at $25^{\circ} \mathrm{C}$ for $10 \mathrm{~min}$. Then, ANS (30 $\left.\mu \mathrm{M}\right)$ was added in the samples and incubated for $30 \mathrm{~min}$ at $25^{\circ} \mathrm{C}$. The fluorescence spectra were recorded in the range of 430 $520 \mathrm{~nm}$ using $350 \mathrm{~nm}$ as an excitation wavelength. The fluorescence spectra of plumbagin (0$120 \mu \mathrm{M}$ ) was also recorded as a blank. The change in fluorescence at $475 \mathrm{~nm}$ was calculated by subtracting blank from the respective data sets. A dissociation constant of the binding interaction of plumbagin with WT-FtsZ and with R191A-FtsZ was determined from the fluorescence change data as described previously ${ }^{1}$. 


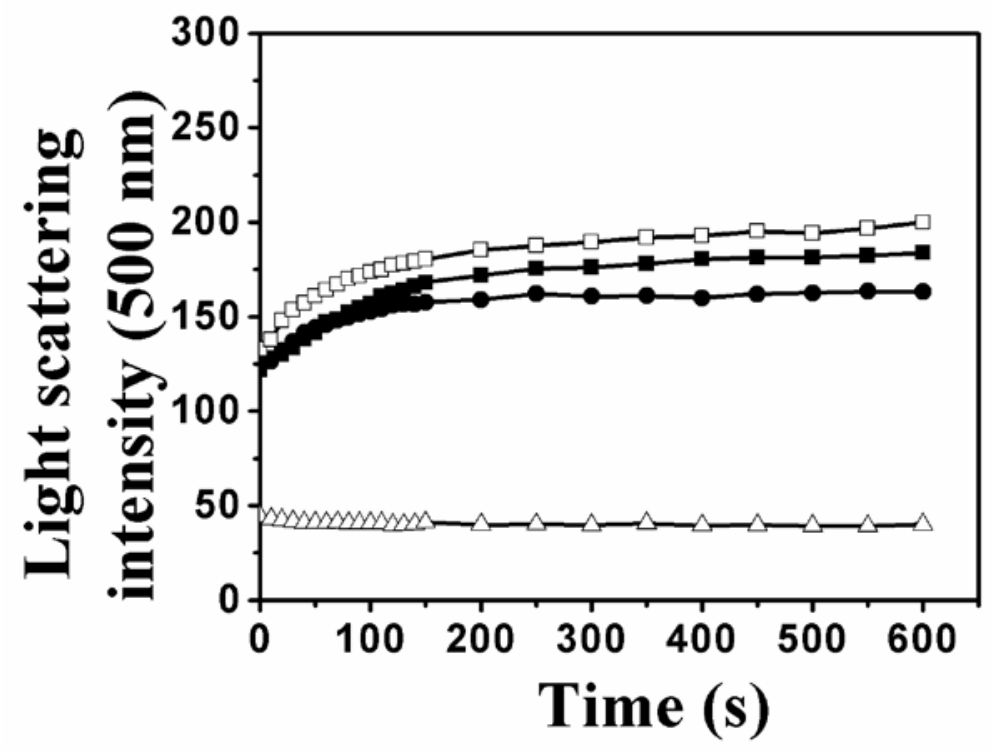

Fig. S2. The effect of plumbagin on the assembly kinetics of R191A-FtsZ. R191A-FtsZ (12 $\mu \mathrm{M}$ ) was incubated without ( $\square$ ) or with $20(\boldsymbol{\bullet})$ and $40 \mu \mathrm{M}(\bullet)$ plumbagin for 15 min on ice and then, the assembly kinetics was monitored by adding $1 \mathrm{mM} \mathrm{GTP}$ at $37^{\circ} \mathrm{C}$. The light scattering intensity of buffer $(\Delta)$ [25 mM PIPES (pH 6.8), $50 \mathrm{mM} \mathrm{KCl}$ and $10 \mathrm{mM} \mathrm{MgCl}_{2}$ ] was also monitored as a blank. 
Wt-FtsZ simulation-1

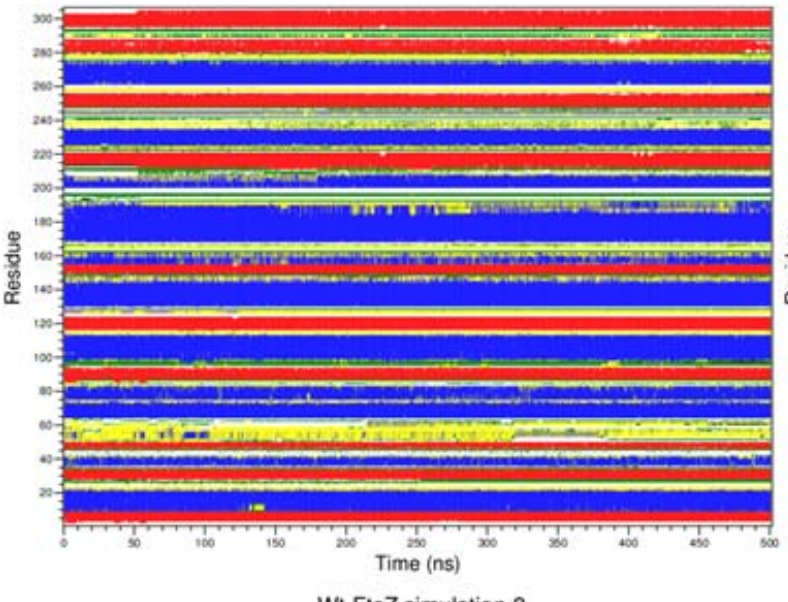

Wt-FtsZ simulation-2

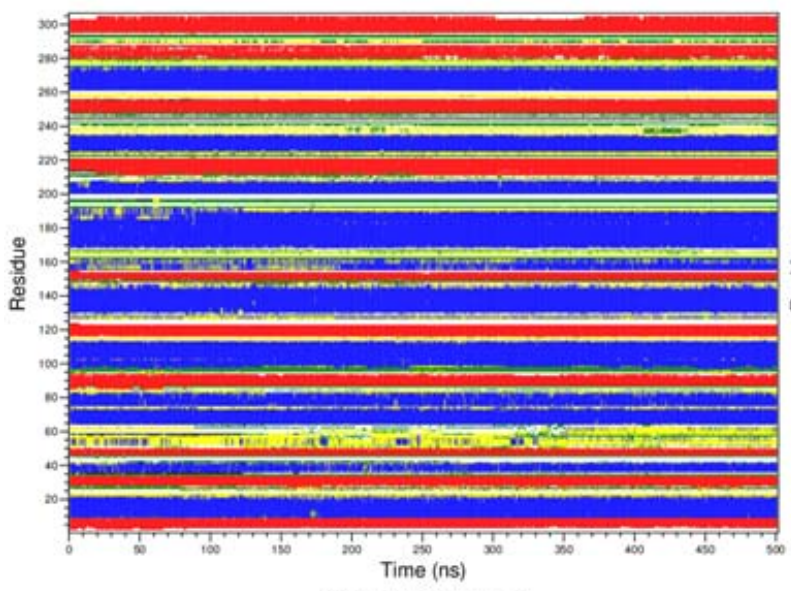

Wt-FtsZ simulation-3

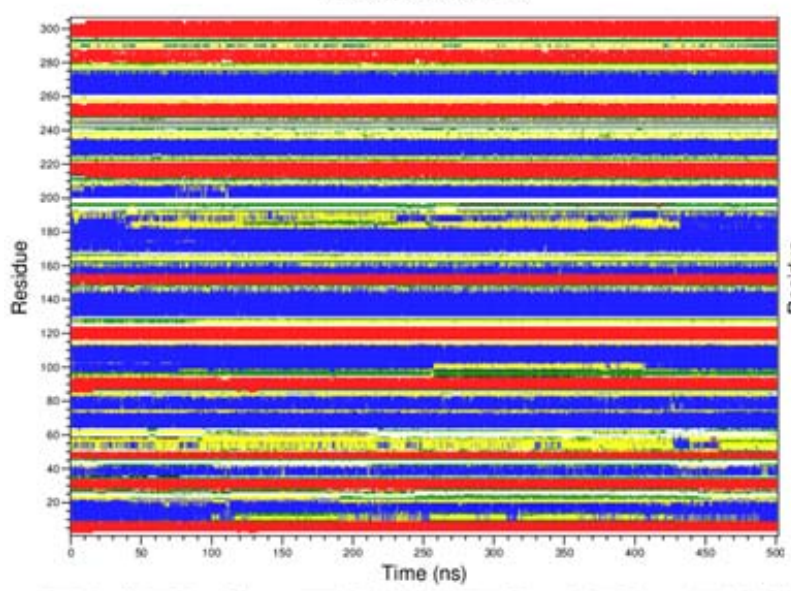

Coil $\square$ B-Sheet $\square$ B-Bridge $\square$ Bend $\square$ Turn $\square$ A-Helix
R191A-FtsZ simulation-1

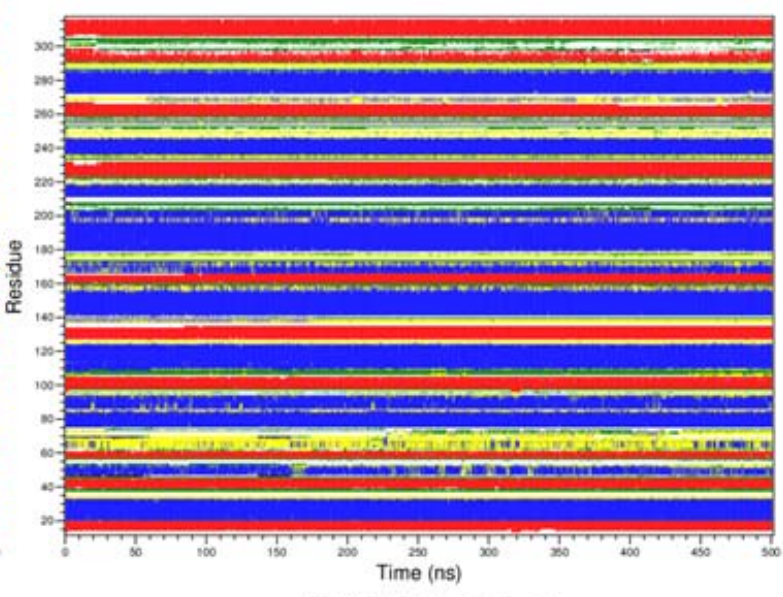

R191A-FtsZ simulation-2

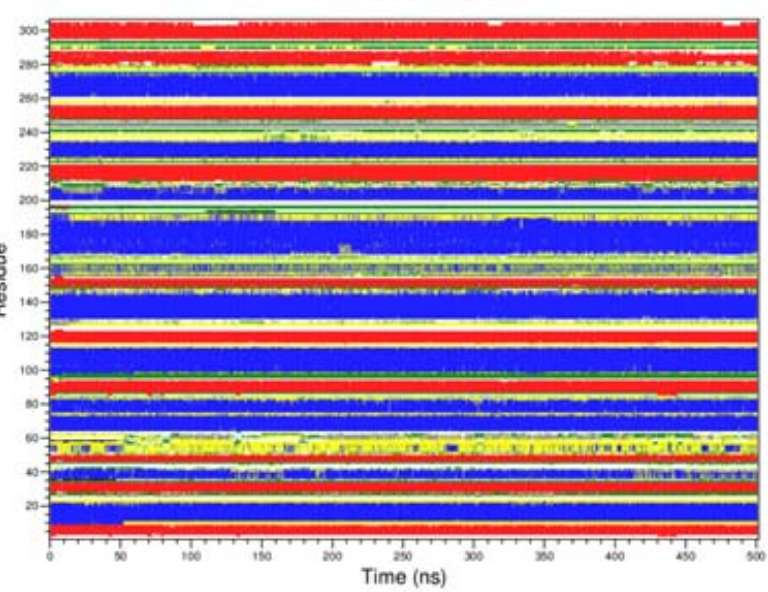

R191A-FtsZ simulation-3

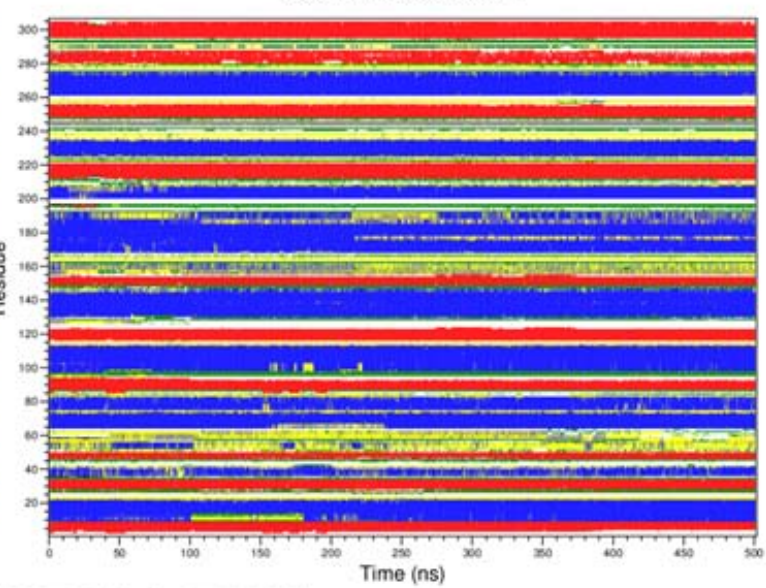

5-Helix $\square$ 3-Helix

Fig. S3: Secondary structures of WT-FtsZ and R191A-FtsZ. Molecular dynamics simulations of WT-FtsZ and R191A-FtsZ generated 20,000 structures in one trajectory. For each structure, secondary structure was calculated using DSSP tool from GROMACS package. On the y-axis secondary structure of each residue is shown and x-axis shows how the secondary structure of each residue evolved during the course of the simulation. H5-helix: residues 179203 and T7-loop: residues 204 to 210. 


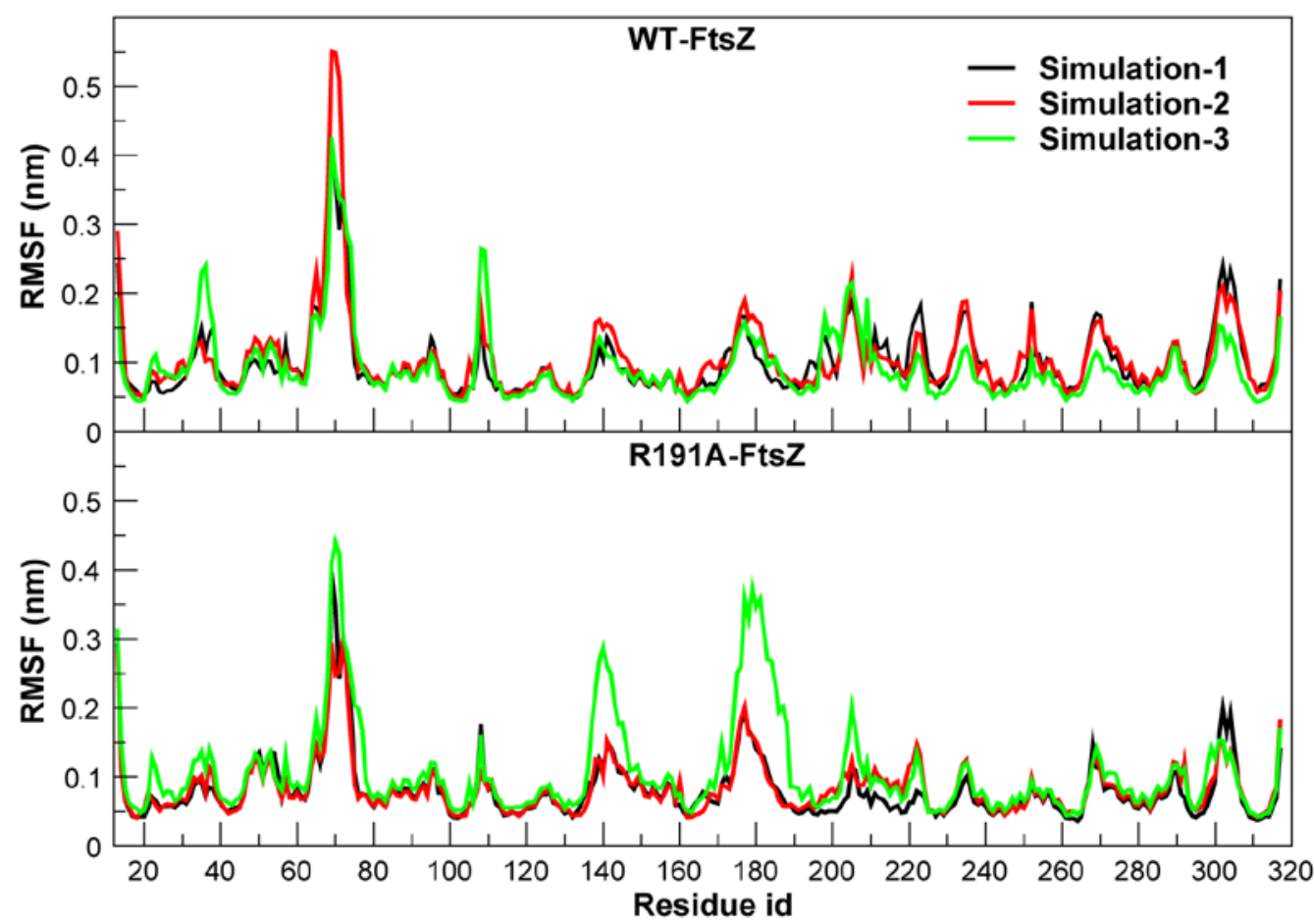

Fig. S4: Root mean square fluctuation analysis of WT-FtsZ and R191A-FtsZ

simulations. Root mean square fluctuation analysis was performed to identify regions of structural change. Three simulations each for the WT-FtsZ and R191A-FtsZ that were performed are shown in black, red, and green. 

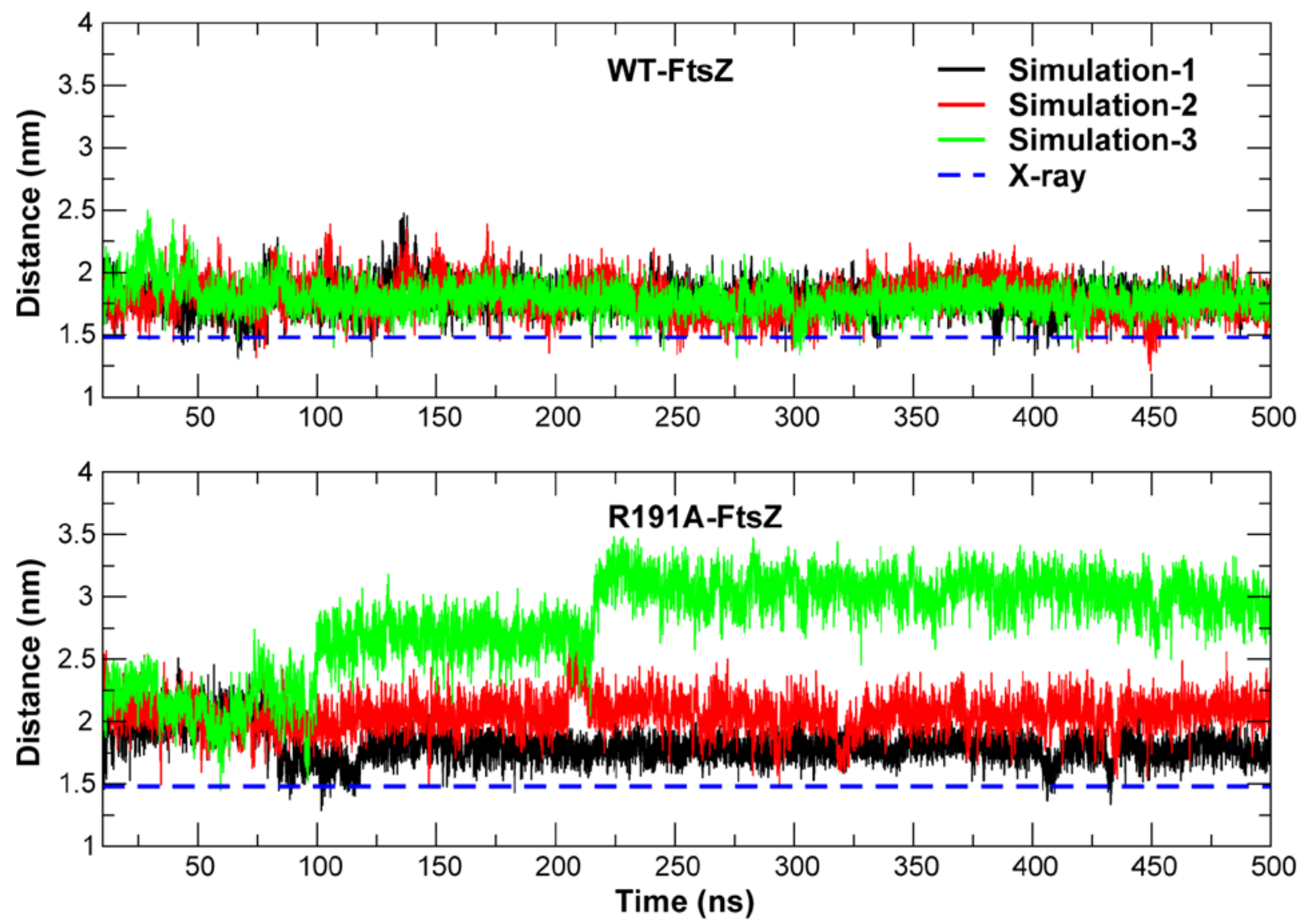

Fig. S5: Distance plot between the residue F138 and N176 C-alpha atoms in WT-FtsZ and R191A-FtsZ simulations. 
Table S1. The role of different domains of FtsZ.

\begin{tabular}{|c|c|}
\hline Domian or residues of FtsZ & Function \\
\hline $\begin{array}{l}\text { 1. N-terminal domain and C-terminal } \\
\text { domain of T. maritima FtsZ }\end{array}$ & $\begin{array}{l}\text { 1. Both domains can fold } \\
\text { independently into functional tertiary } \\
\text { structure. }\end{array}$ \\
\hline $\begin{array}{l}\text { 2. Poorly conserved last } 6 \text { residues } \\
\text { (NRNKRG) of } B \text {. subtilis FtsZ }\end{array}$ & $\begin{array}{l}\text { 2. These residues are essentially } \\
\text { required to promote the high degree of } \\
\text { lateral interactions between FtsZ } \\
\text { polymers. The change in this region of } \\
\text { FtsZ produces significant defect in cell } \\
\text { division in vivo. }\end{array}$ \\
\hline $\begin{array}{l}\text { 3. a) N1-FtsZ (1-178 residues) and } \\
\text { N2-FtsZ (1-204 residues) of B. subtilis } \\
\text { FtsZ } \\
\text { b) C1-FtsZ ( } 205-366 \text { residues), C2- } \\
\text { FtsZ (176-366 residues) and C3-FtsZ } \\
\text { (176-382 residues) of B. subtilis FtsZ }\end{array}$ & $\begin{array}{l}\text { 3. a) Both N-domains have ability to } \\
\text { polymerize and form filamentous } \\
\text { polymers independently. }{ }^{4} \\
\text { b) These C-domains cannot form } \\
\text { polymers and also inhibited the } \\
\text { Polymerization of FL-FtsZ. }{ }^{4}\end{array}$ \\
\hline $\begin{array}{l}\text { 4) Mutations of Asn207, Asp209, and } \\
\text { Asp212 in the T7 loop of EcFtsZ }\end{array}$ & 4) Severely affected GTP hydrolysis. ${ }^{5}$ \\
\hline $\begin{array}{l}\text { 5) H7 helix of Methanococcus } \\
\text { jannaschi FtsZ }\end{array}$ & $\begin{array}{l}\text { 5) H7-helix maintains communication } \\
\text { between } \mathrm{N} \text {-and C-terminal domain, } \\
\text { and bending of it can regulate } \\
\text { assembly/disassembly of FtsZ. }^{6}\end{array}$ \\
\hline
\end{tabular}

Table S2. Comparison of root mean square deviation of backbone atoms of entire protein and only helices H4 and H7.

\begin{tabular}{|c|c|c|c|c|c|c|}
\hline \multirow{2}{*}{} & \multicolumn{2}{|c|}{ Simulation-1 } & \multicolumn{2}{c|}{ Simulation-2 } & \multicolumn{2}{c|}{ Simulation-3 } \\
\cline { 2 - 6 } & $\begin{array}{c}\text { Entire } \\
\text { protein } \\
\end{array}$ & $\begin{array}{c}\text { H5 \& } \\
\text { H7(nm) } \\
(\mathrm{nm})\end{array}$ & $\begin{array}{c}\text { Entire } \\
\text { protein(nm) }\end{array}$ & $\begin{array}{c}\text { H5 \& } \\
\text { H7(nm) }\end{array}$ & $\begin{array}{c}\text { Entire } \\
\text { protein(nm) }\end{array}$ & $\begin{array}{c}\text { H5 \& } \\
\text { H7(nm) }\end{array}$ \\
\hline $\begin{array}{c}\text { WT-FtsZ } \\
\text { R191A- } \\
\text { FtsZ }\end{array}$ & $0.16 \pm 0.02$ & $0.19 \pm 0.02$ & $0.19 \pm 0.04$ & $0.2 \pm 0.03$ & $0.17 \pm 0.02$ & $0.18 \pm 0.03$ \\
\hline
\end{tabular}

Average \pm Standard deviation 


\section{Movie S1: The MD simulation of R191A-FtsZ and WT-FtsZ.}

Movie is attached as FtsZ_HelixTilt.vlc file.

\section{References:}

(1) Bhattacharya, A., Jindal, B., Singh, P., Datta, A., and Panda, D. (2013) Plumbagin inhibits cytokinesis in Bacillus subtilis by inhibiting FtsZ assembly--a mechanistic study of its antibacterial activity. FEBS J. 280, 4585-4599.

(2) Martín-Galiano, A. J., Buey, R. M., Cabezas, M., and Andreu, J. M. (2010) Mapping flexibility and the assembly switch of cell division protein FtsZ by computational and mutational approaches. J. Biol. Chem. 285, 22554-22565.

(3) Scheffers, D.-J., de Wit, J. G., den Blaauwen, T., and Driessen, A. J. M. (2002) GTP hydrolysis of cell division protein FtsZ: evidence that the active site is formed by the association of monomers. Biochemistry 41, 521-529.

(4) Jindal, B., and Panda, D. (2013) Understanding FtsZ assembly: cues from the behavior of its N- and C-terminal domains. Biochemistry 52, 7071-7081.

(5) Buske, P. J., and Levin, P. A. (2012) Extreme C terminus of bacterial cytoskeletal protein FtsZ plays fundamental role in assembly independent of modulatory proteins. J. Biol. Chem. 287, 10945-10957.

(6) Oliva, M. A., Trambaiolo, D., and Löwe, J. (2007) Structural Insights into the Conformational Variability of FtsZ. J Mol Biol. 373, 1229-1242. 